\title{
LA FENOMENOLOGÍA DE HUSSERL COMO FUNDAMENTO FILOSÓFICO PARA LA TEOLOGÍA
}

\section{THE PHENOMENOLOGY OF HUSSERL AS A PHILOSOPHICAL FOUNDATION FOR THEOLOGY}

\author{
Prof. Dr. Francisco-Javier Herrero-Hernández ${ }^{1}$ \\ Universidad Pontificia de Salamanca, España
}

\begin{abstract}
Resumen: El principal objetivo de este trabajo es lograr una comprensión de la la fenomenología de Husserl como fundamento filosófico para la teología. Sostiene, en primer lugar, la idea de que la teología y la filosofía hacen algo más que converger. Profundiza, en segundo lugar, en la conexión entre fenomenología y teología, así como en la concepción

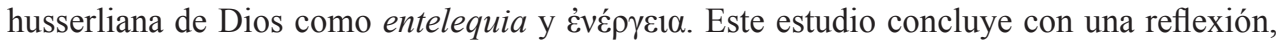
en tercer lugar, sobre la posibilidad de elaborar una teología de inspiración fenomenológica. La tesis que se defiende es que la fenomenología supone para la teología, literalmente, una auténtica Herausforderung, es decir, una provocación que desde fuera interpela a un discurso, el teológico, que muchos juzgaban ya agotado y en riesgo de extinción. En las conclusiones se detallan las principales aportaciones que la fenomenología hace a la teología.
\end{abstract}

Descriptores: Fenomenología $\cdot$ Teología $\cdot$ Razón $\cdot \mathrm{Fe} \cdot$ Dios $\cdot$ Husserl

\begin{abstract}
The main objective of this work is to achieve an understanding of Husserl's phenomenology as philosophical foundation for theology. It sustains, in the first place, that theology and philosophy do more than converge. It deepens, in second place, in the connection between phenomenology and theology, as well as in the Husserlian conception

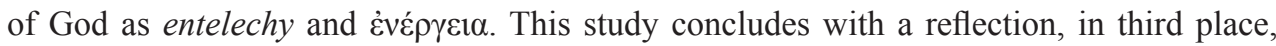
on the possibility of elaborating a theology from the phenomenological inspiration. The thesis that is defended is that phenomenology means for theology, literally, an authentic Herausforderung, that is to say, a provocation that from outside interpellates a discourse, the theological one, that many judged already exhausted and at risk of extinction. In the conclusions are detailed the main contributions that the phenomenology makes to theology.
\end{abstract}

Keywords: Phenomenology $\cdot$ Theology $\cdot$ Reason $\cdot$ Faith $\cdot$ God $\cdot$ Husserl

\section{Teología y Filosofía: algo más Que conVerger.}

Teología y Filosofía, a pesar de sus innegables diferencias, comparten no solo una historia común, sino un mismo destino. La razón de esta especie de mutua pericóresis radica en que ambas disciplinas se plantean las mismas preguntas por el

\footnotetext{
${ }^{1}$ Facultad de Filosofía. E-mail: fjherrerohe@upsa.es
} 
conocer, el deber, el esperar y, de manera compendiada, por la vida del hombre en su verdad más última y definitiva. ${ }^{2}$ Esta relación, sin embargo, no ha resultado ser siempre demasiado armoniosa, disputándose a cada paso de su común historia la preeminencia de una sobre la otra. Las teorías se dividen, a este respecto, entre las que afirman una identidad esencial (Wesensidentität), total o parcial, y aquellas que proponen una distinción esencial (Wesensverschiedenheit) entre ambas realidades. ${ }^{3}$

Más allá de las evidentes afinidades y de las contrastadas desavenencias habidas en su pluridireccional devenir histórico, lo cierto es que tanto la naciente filosofía como la primitiva teología (cristiana) estuvieron destinadas desde un principio a algo más que a converger. ${ }^{4} \mathrm{El}$ dato irrefragable es, en efecto, que sus caminos iniciales mostraron siempre el mismo interés por la verdadera figura, la auténtica naturaleza de la realidad divina. ${ }^{5}$ Frente a las afirmaciones tradicionales de las religiones sobre los muchos dioses instituidos (thései theoi), la fe cristiana sintió la necesidad, casi natural, de vincularse con aquella primera y crítica ilustración griega que acentuaba la verdad del único dios natural (phýsei theós). ${ }^{6}$ Desde entonces, la relación entre teología y filosofía ha atravesado, en efecto, momentos de oposición, de identidad y de mutua subordinación. ${ }^{7}$ Pero a lo que nunca renunciaron ambas disciplinas fue a la convicción, mantenida prácticamente de consuno, de la correlación insoslayable entre el concepto de la totalidad de lo real o mundo y la idea misma de Dios. Y es precisamente esa

\footnotetext{
${ }^{2}$ Véase (Kant, Logik. Ein Handbuch zu Vorlesungen (Jäsche), AA, IX, p. 25). La religión según Kant surgiría, pues, como respuesta a la pregunta sobre qué me está permitido esperar. Es la pregunta por el futuro, es decir, la historia y el sentido de la existencia humana. Viene a prolongar la pregunta del deber moral pues ambas, la del deber y la de la pertinencia en poder esperar, cubren el arco de la conducta humana. La pregunta se desdobla en realidad en dos partes. La filosofía de la historia investigaría la esperanza de la libertad externa: el derecho; y la filosofía de la religión, indagaría la esperanza de la libertad individual: la virtud o la moralidad.
}

${ }^{3}$ Se trata de la clásica distinción establecida por Max Scheler (Scheler, 2007; GW 5, p. 126). Para la relación entre Teología y Filosofía véase (Pannenberg, 1996), (Lacoste, 1998, pp. 901-906 y 1126-1132 con bibliografia), (Rohls, 2002), (Delgado \& Vergauwen, 2003), (González de Cardedal, 2003, pp. 5-31), (Dietzch \& Frigo, 2006), (Hailer, 2006), (Müller, 2006), (Caputo, 2006), (Capelle-Dumont, 2009a, 2009b, 2010 y 2011), (Falque, 2013), (Dalferth, \& Hunziker, 2014).

${ }^{4}$ La expresión "más que converger" procede de E. Levinas y no puede significar otra cosa que el dato de la posible y fundamental coincidencia entre teología y filosofía. El presupuesto de Levinas es que la filosofía, frente a lo que habitualmente suele afirmarse, no habría sido nunca esencialmente atea y, en consecuencia, no existiría tampoco necesidad alguna de emprender la pretendida conciliación porque sencillamente no existiría ninguna rivalidad insalvable entre ellas. La filosofía reposaría, según él, sobre una experiencia prefilosófica, una especie de segunda escritura que subyace oculta en su interior. Filosofar no sería en realidad sino descifrar esa escritura escondida como si de un palimpsesto se tratara. Véase (Levinas, 1995, p. 148; 1988, p. 155). También Husserl hablará de una esencia coincidencia in infinitum entre filosofía y teología. Véase (Husserl, 2013, aquí p. 260).

${ }^{5}$ Con Werner Jaeger, en efecto, nos hemos acostumbrado a hablar de los primeros pensadores griegos como verdaderos teólogos. Véase (Jaeger, 1953 y 1965) y (Holscher 1968).

${ }^{6}$ Véase (Jaeger, 1953, pp. 10s.).

${ }^{7}$ Un buen resumen de las distintas formas de presentar esta relación se puede encontrar en (Cabria, 2008, pp. 164ss.). 
común orientación hacia el Todo como lo más verdadero ${ }^{8}$ la que echamos de menos al ver hoy cómo la filosofía -y no sólo la teología- ha desistido también, en gran medida, de su tarea de vérselas con el todo del hombre, del mundo y de Dios. ${ }^{9}$

El problema es que en nuestro tiempo escasean, por un lado, los filósofos que se atrevan a defender lo que Hegel aún sostenía cuando afirmaba que «Dios es el objeto único de la filosofía». ${ }^{10}$ Pero al desaparecer Dios del núcleo de todo pensar, éste declina poder llegar al fondo de sí mismo. Cuando la filosofía no desemboca en la trascendencia acaba, en efecto, decapitándose a sí misma como pensamiento según la conocida sentencia de Adorno. ${ }^{11} \mathrm{Y}$ la teología, por su parte, terminará vaciándose también de contenido si concede el monopolio de la interpretación de la realidad a la conciencia profana y se repliega sobre la interioridad pura del sentimiento como de nuevo advirtiera ya el citado pensador alemán. ${ }^{12} \mathrm{El}$ discurso teológico debería ser, en consecuencia, más autoconsciente, ${ }^{13}$ es decir, tendría que vincularse aún más a la reflexión sobre la globalidad para presentarse así con significatividad real para el hombre actual. ${ }^{14}$

Es sabido que una de las empresas que concitaron los mejores esfuerzos de muchos de los grandes pensadores fue precisamente la de intentar armonizar la "vieja antítesis"15 entre teología y filosofía. Conocemos también los recelos mutuos y las desarmonías históricas de estas dos magnitudes que, como "agua y aceite", ${ }^{16}$ han estado determinado gran parte de la historia de Occidente hasta el punto de terminar

${ }^{8}$ Fue Hegel el que dejó escrito en el Prólogo a su Fenomenología del Espíritu que "Lo verdadero es el Todo: Das Wahre ist das Ganze" (Hegel, 1986, p. 24).

${ }^{9}$ Véase (Pannemberg, 1996, pp. 18s y 1988, pp. 7-20). Con su bellísima "Meditación sobre la palabra 'Dios", Karl Rahner nos ayudó a ver las consecuencias que conllevaría la desaparición del término "Dios". Véase (Rahner, 1976, pp. 54-61).

10 “... es el punto de partida y la meta final de todo; todo parte y retorna a él" Incluso la filosofía no tiene otro objeto más que Dios: "Dios es así el único objeto de la filosofía, su tarea consiste en ocuparse de él, en reconocerlo todo en Él, referirlo todo a Él así como en derivar de Él todo lo particular y en justificarlo todo sólo en la medida en que surge de Él, se mantiene en conexión con Él, vive de su irradiación y tiene su alma allí en Él. La filosofía es, por tanto, teología y ocuparse de ella o más bien en ella es para sí culto divino" (Hegel, 1983, pp. 57 y 85 respectivamente).

${ }^{11}$ Véase (Adorno, 1996, p. 393). Wilhelm Weischedel, por su parte, dedicó una extensa obra a mostrar las consecuencias que este abandono había acarreado a la filosofía. En su clásico Der Gott der Philosophen (ahora en un único volumen) analiza hasta qué punto la filosofía occidental se convirtió en una doctrina filosófica sobre el Dios cristiano y cómo la renuncia de esta tarea ha conllevado la crisis misma de la filosofía. Véase (Weischedel, 1998, p.218) y (Torres, 2000, p. 208).

${ }^{12}$ Véase (Hegel, 1983, pp. 25 y 23).

${ }^{13}$ Klaus Müller, profesor en Münster de 'Cuestiones filosóficas fundamentales para la teología' es, sin duda, uno de los autores más pródigos en el estudio de las relaciones entre filosofía y teología. Fruto de su dedicación a este tema es su valiosísima y voluminosa obra Glauben, Fragen, Denken (Müller, 2006, 2008 y 2010a). Véase también (Müller, 2010b).

${ }^{14}$ Véase (González de Cardedal, 2008).

${ }^{15}$ (Hegel, 1989, p. 60).

${ }^{16}$ (Kant, 1969, p. 27). 
repercutiendo profundamente tanto en la conducta humana, como en la moral e incluso en las mismas bases políticas de sus sociedades. Lo cierto es que cuando la incompatibilidad entre el creer y el saber logra imponerse frente a esos otros impulsos sintetizadores, la convivencia comienza a supurar intolerancia y violencia. La superación de la escisión entre razón y fe mediante un diálogo maduro que respete las inequívocas e irrenunciables peculiaridades de cada una de ambas instancias es, a fecha de hoy, un problema aún por resolver en el actual debate cultural entre civilizaciones y religiones. ${ }^{17}$

Renuncio aquí, por supuesto, a la tarea de trazar el cuadro completo del fenómeno de la separación moderna entre teología y filosofía. Pero si queremos avanzar hacia la esencia misma de las cosas -sin detenernos por tanto en el proceloso y nada fácil itinerario histórico de este alejamiento- llegará un momento en el que no podamos contentarnos, en absoluto, con la simple descripción de este divorcio mortífero entre "la reflexión cultivada" y el "sentimiento religioso" excluyéndose mutuamente. Ante el cuadro de esta escisión surge, más bien, la imperiosa necesidad de llevar a cabo una reconciliación nueva entre religión y razón, entre teología y filosofía a través de un proceso de aprendizaje complementario (komplementärer Lernprozess) como viene proponiéndonos desde hace ya algún tiempo Jürgen Habermas. ${ }^{18}$ Pues bien, el movimiento fenomenológico iniciado por Edmund Husserl tiene la virtud de haber procurado la posibilidad de convertirse en el aliado filosófico, nada casual como veremos, de una teología que -desde Kant, al menos- permanecía a la espera de una filosofía que no se redujese a ser la mera repetición de la metafísica clásica y propusiera, como es el caso, la cuestión de Dios en cuanto pregunta por la verdad misma de las cosas. Sobre esta real posibilidad de inspiración que para la teología está suponiendo la fenomenología es de lo que pretendemos reflexionar en los siguientes apartados.

\section{La fenomenología de Husserl y la teología: Dios como ENTELEQUIA Y \&́vé́ $\rho \varepsilon l \alpha$.}

Dominique Janicaud nos malacostumbró a contemplar la posibilidad de que la fenomenología, al menos en el área francesa, hubiera experimentado una especie de giro hacia la teología. ${ }^{19}$ Sus provocadores escritos sobre ese supuesto

\footnotetext{
${ }^{17}$ He tenido oportunidad de pronunciarme al respecto en (Herrero-Hernández, 2016b, pp. 4-22).

18 (Habermas, 2005, p. 31). En contra de la posición habermasiana sobre la postsecularidad, véase (Flores D'Arcais, 2008 y 2014).

${ }^{19}$ Véase (Janicaud, 1990). La denuncia de Janicaud se dirige, especialmente, contra la «restauración metafísica (y teológica)» llevada a cabo por Levinas y en la que, de hecho, «la fenomenología ha sido tomada como rehén por una teología que no quiere decir su nombre» (ibíd., p. 31). En este sentido, Totalidad e infinito (Levinas, 1961) representaría, según él, la primera gran obra de la filosofía francesa que habría asumido de manera explícita el giro teológico. Para un acercamiento al debate provocado por estos escritos véase (Herrero-Hernández, 2016a, pp. 239ss.).
} 
giro abrieron la puerta para que un sinfín de detractores y adeptos comenzaran a manifestar su más radical repulsa o su ferviente aceptación, según el caso, ante una denuncia como la suya que, en el fondo, terminaba planteando un serio interrogante sobre la posibilidad misma de la fenomenología. ${ }^{20}$ Cabe, sin embargo, invertir la situación y replantear el asunto de otra manera. Podemos cuestionar, por ejemplo, si este supuesto giro no haya sido tal en realidad, es decir, podemos reflexionar sobre la posibilidad también de que la misma fenomenología hubiera estado, desde un principio, vinculada estrechamente a la teología. ${ }^{21}$ En este sentido, la fenomenología no se habría desviado de su destino, sino que habría respondido fielmente a su más auténtica e interna virtualidad: la de poder referirse, en su pura fenomenalidad, a la trascendencia primordial y tematizar la aparición de lo absoluto identificado, seguidamente, con el sujeto absoluto en cuanto principio de todo fenómeno. ${ }^{22} \mathrm{Y}$ habría sido el propio Husserl, además, el encargado ya de haber abierto esta posibilidad de ir más allá de la objetividad constituible. Como es sabido, su interés por lo inobjetivable fue haciéndose más patente a partir, sobre todo, de sus investigaciones de 1905 sobre La conciencia interna del tiempo (Husserl, 1969), en las que el maestro exploraba una intencionalidad más originaria, no objetivante $y$, por tanto, "pasiva". 23

La cuestión no es nueva y contamos con numerosos estudios consagrados a esclarecer la posibilidad de esta inicial conexión entre la fenomenología husserliana y la teología. ${ }^{24}$ Todos ellos coinciden en mostrar el interés prematuro con el que maestro abordó la cuestión de Dios y se apoyan, sobre todo, en los famosos manuscritos con la signatura de archivo E en el Nachlass que corresponden al

\footnotetext{
${ }^{20}$ Janicaud avivó aún más la polémica de su primer escrito publicando algunos años más tarde otro beligerante ensayo en el que hablaba ya del estallido mismo de la fenomenología. Véase (Janicaud, 1998). Su impresión entonces es que en los últimos tiempos habríamos asistido a una espectacular eclosión de la fenomenología en múltiples y divergentes orientaciones, y la pregunta que nos tendríamos que hacer es si este estallido significa, realmente, la imposibilidad de la fenomenología como algún autor ya se había adelantado a sugerir. En realidad, el giro teológico de la fenomenología contemporánea no constituiría sino un síntoma elocuente de su intrínseca imposibilidad. Véase (Alliez, 1995), (Benoist, 1994), (Harr, 1999), (Escoubas \& Waldenfels, 2000, pp. 175-213).

${ }^{21}$ Son clásicos los trabajos de (Van Breda, 1949, pp. 1210-1212) y (Strasser, 1959).

${ }^{22}$ Véase (Alliez, 1995, p. 63).

${ }^{23}$ Véase (Marion, 1992, pp. 186s.) y (Alliez, 1995, p. 60).

${ }^{24}$ Entre la bibliografía más actualizada sobre el problema de Dios en Husserl y la conexión inicial entre fenomenología y teología, destaco los trabajos de (Ales Bello, 1985, 1995 y 2005), (Laycock \& Hart, 1986), (Hart, 1990), (Iribarne, 1994), (Lo, 2008), (Housset, 2010), (Held, 2010), (Walton, 2012) y (Copertino, 2014). Contrario a este tipo de interpretaciones, Jocelyn Benoist cuestiona que Husserl haya ido más allá de la ontoteología. Se apoya, principalmente, en el manuscrito F 24 (Formale Ethik und Probleme der praktischen Vernunft) donde Dios, a fin de cuentas, vuelve a ocupar el papel reservado para él desde Aristóteles. Véase (Benoist, 1991, p. 439). De manera parecida, Mikel Dufrenne se había referido ya a las posibles ambigüedades que parecen mostrar tanto Husserl como Heidegger con relación a la herencia teológica. Véase (Dufrenne, 1973, pp. 7-57).
} 
tratamiento en forma preliminar que Husserl hizo de los temas sobre la filosofía de la religión y el problema de Dios. El volumen XLII de Husserliana ha abierto el acceso a todo este amplio caudal de documentos, la mayoría inéditos, sobre los Grenzprobleme (Husserl, 2013, pp. 137-263.). Las fechas de estos textos van de 1908 a 1937 y ponen de manifiesto su continuada dedicación con los problemas últimos de la metafísica. Es cierto que en esta documentación no vamos a encontrar un tratamiento sistemático sobre el problema de Dios, pero lo que sí podremos es hallar algunos pasajes de su análisis fenomenológico que abren la posibilidad de una vía de investigación metodológicamente atea hacia lo Absoluto. ${ }^{25}$ En todo caso, este acceso a la divinidad tendrá que seguir con fidelidad los principios fundamentales que guían a la fenomenología. Dios será considerado siempre desde lo que es para mí, es decir, a partir de mi propio rendimiento de conciencia (aus meiner eigenen Bewusstseinsleistung). ${ }^{26}$

La idea de Dios que Husserl siempre ha admitido viene determinada por su concepción teleológica del mundo y del ethos humano. Por una parte, los procesos naturales parecen responder a una evidente finalidad ordenada y a esta misma certeza llegamos cuando observamos el sentido último al que tiende el ascendente ethos humano. El maestro plantea, por tanto, la posibilidad de una meta o de un fin absoluto y universal, es decir, de un telos. Pero este telos así concebido coincide básicamente con la idea de Dios. Se trata de esa "admirable teleología" que acaba llevándonos hasta la teología y que él ha tildado habitualmente como una "vía atea" hacia Dios. Y lo mismo sucede cuando se adopta la perspectiva del desarrollo ético de la humanidad. Aquí, Husserl no parece ir más lejos de Kant cuando propone la idea de Dios como telos práctico ideal, fin ideal o entelequia práctica. La existencia del imperativo categórico "exige de mí una acción orientada a un universal ascenso de valores del mundo humano" (Husserl, 2013, p. 168). Y lo que el maestro sostiene finalmente es que para que esta exigencia ética sea absoluta, o sea, para que ella sea válida para mí, tendré que considerar la necesidad de creer también en el sentido del mundo.

Lo que a nosotros nos interesa ahora es destacar que, a la altura, al menos, de la Crisis de las Ciencias europeas ${ }^{27}$, el maestro habría alcanzado el convencimiento pleno de que el problema filosófico de Dios es el que alberga, de manera evidente, el problema de la razón "absoluta". Para el Husserl maduro, el problema filosófico de la razón "absoluta" está contenido, en consecuencia, en la idea de Dios en cuanto fuente teleológica de toda razón y del "sentido"

\footnotetext{
${ }^{25}$ Me he ocupado de esta cuestión en (Herrero-Hernández, 2016a, pp. 233-243).

${ }^{26}$ (Husserl, 1974, p. 99).

${ }^{27}$ (Husserl, 1954).
} 
de un mundo morfológicamente ordenado: ${ }^{28}$ Pues bien, es precisamente esta especie de teleología universal de la razón (ontológica y ética) la que le permite elaborar, de manera ciertamente preliminar, la posibilidad de una teología filosófica inspirada en la fenomenología y en la que Dios se presenta finalmente como quien actúa a modo de telos en la existencia de los sujetos, constituyendo así el sentido de la vida: "Gott als Entelechie, Gott als ćvé $\gamma \gamma \varepsilon l \alpha "$ (Husserl, 2013, p. 168).

No pretendo ahora, naturalmente, adentrarme en el problema del acceso a Dios tal como lo ha venido planteando Husserl a lo largo de su itinerario intelectual. Mi objetivo en este momento se centra en la consideración filosófica del problema, es decir, la cuestión de la relación entre la fenomenología y la teología en cuanto problema filosófico tal como el maestro la habría apenas diseñado. ${ }^{29}$ La filosofía ha de ser comprendida, según él, como la obra de una razón plenamente autónoma, como "autorreflexión de la humanidad sobre aquello que para ella posee el valor de Absoluto, sobre aquello que da verdad y sentido a su ser mundano como vida-en-el-mundo" (Husserl, 1989, p. 234). A la luz de lo dicho, no hay duda de que Husserl debe ser considerado, evidentemente, como un hombre de la Aufklärung en el mejor sentido de la palabra, es decir, persuadido de que nada en el universo permanece cerrado ni posee fuerza suficiente para resistir la iluminación de la razón. El programa que la razón ha de llevar a cabo es el de tratar de escudriñar hasta el rincón más último y oscuro de la realidad, aun cuando esto suponga un proceso indefinido de trabajo. ${ }^{30}$ Y si este es el programa de la filosofía en general, la fenomenología deberá cumplir una expectativa aún más pretenciosa si cabe, tratando de hacer posible, desde el punto de vista ético-religioso, una vida regida por normas puramente racionales y donde los filósofos acaben siendo considerados como "funcionarios de la humanidad". ${ }^{31}$ En el próximo apartado trataré de concretar si la propuesta husserliana que acabo de exponer someramente ofrece la posibilidad real de elaborar una teología de inspiración fenomenológica.

${ }^{28}$ «Das Gottesproblem enthält offenbar das Problem der „absoluten Vernunft als der teloeologischen Quelle aller Vernunft in der Welt, des „Sinnes“ der Welt» (Husserl, 1954, § 3, 7). Véase (Strasser, 1959, p. 136).

${ }^{29}$ Véase el texto $\mathrm{n}^{\mathrm{o}} 20$, de la parte III del volumen XLII de Hua. que lleva significativamente el siguiente título: "Filosofía y teología: Su relación como problema filosófico. Teología filosófica como culminación de la filosofía y teología confesional como continuación de la filosofía y empleada como herramienta" (Husserl, 2013, pp. 259-263).

${ }^{30}$ Véase (Fink, 1968, p. 207).

${ }^{31}$ Véase (Husserl, 1987 y 1976, pp. 3 y 15 respectivamente). 


\section{HACIA UNA TEOLOGÍA DE INSPIRACIÓN FENOMENOLÓGICA}

La fenomenología viene ofreciendo a la teología la oportunidad que buscaba azarosamente desde Kant, a saber: La necesidad de proveer a su reflexión de un solar filosófico sólido desde el que seguir manteniendo y desarrollando sus afirmaciones con plausibilidad racional y a la altura intelectual del momento que le ha tocado vivir. No se trata ahora de exponer el ser y el quehacer de la teología hoy, ${ }^{32}$ pero es innegable que la fenomenología está suponiendo para ella, literalmente, una auténtica Herausforderung, es decir, una provocación que desde fuera interpela a un discurso, el teológico, que muchos juzgaban definitivamente agotado y a punto ya de extinción. Tuve ocasión de referirme al problema del vaciamiento de la teología tal como lo había planteado Hegel en su tiempo. Lo que el filósofo alemán nos decía entonces -válido igualmente para nuestro actual momento- era que el proceso de marginalización cultural experimentado por la teología resultaba ser fruto, a su vez, de su erosión intelectual y consecuencia del divorcio entre la conciencia mundana y la religiosa. La teología, en su repliegue subjetivista, se habría abandonado a las "brumas calurosas del fervor que se manifiestan -decía él- totalmente sordas a la música del concepto".33

Si convenimos ahora también nosotros en aceptar la recomendación hegeliana para superar el hiato entre filosofía y teología o razón y fe, parece evidente que el tema último y auténtico del quehacer teológico no podrá ser el de la historia de la salvación, ni la Iglesia o la comunidad, sino Dios. Este ha sido, además, el planteamiento tradicional en los grandes teólogos. En el decir acertado de Santo Tomás de Aquino, por ejemplo, Dios ha de ser el objeto de esta ciencia y también su sujeto. ${ }^{34}$ Pero si esto es así -y dado que de Dios, obviamente, no tenemos ningún conocimiento directo ni podemos alcanzarlo como rendimiento adecuado en nuestra conciencia- la vía para llegar a él tendrá que discurrir necesariamente también al modo filosófico. Y solo manteniéndose en este cauce preciso del pensamiento podrá la teología evitar el riesgo de encallar en el nivel de la imprecisa experiencia

\footnotetext{
${ }^{32}$ Véase (González de Cardedal, 2008).

${ }^{33}$ Hegel introduce una larga reflexión sobre la Ilustración en el capítulo VI de su Fenomenología del Espíritu. Se refiere allí ya a la crisis de la conciencia religiosa que supuso la racionalidad crítica de la Aufklärung. La religión o la fe se presentan entonces como la "Ilustración insatisfecha o la insatisfecha Ilustración", es decir, como una fe que se ha quedado sin contenido, en el vacío. Véase (Hegel, 2009, pp. 674ss.). En las Lecciones de filosofía de la religión arremeterá con dureza contra los intentos de los teólogos de su tiempo buscando pactar a toda costa con el racionalismo deísta, con la exégesis crítica de la Escritura o con la erudición histórica. Véase (Hegel, 1989, pp. 25ss.).
}

${ }^{34}$ Véase Summa theol. I q. 1 a. 7 
religiosa. ${ }^{35}$ Este sería uno de los motivos principales por los que el pensamiento teológico debe vincularse intrínsecamente al modo de cuestionar filosófico como a su método fundamental. La filosofía, como camino independiente del espíritu humano, deberá consecuentemente anteceder a la teología y conservarse siempre como tal, incluso después del acaecer mismo de la revelación. ${ }^{36}$

El lugar de la teología, por tanto, pasa por asumir hoy la tarea de desarrollar una teología más autoconsciente ${ }^{37}$ en el sentido racional del $\sigma \grave{v} \nu \lambda o ́ \gamma \omega,{ }^{38}$ es decir, a partir del programa plenamente actual de la fides quarens intellectum. La teología, en consecuencia, deberá interrogarse también hoy acerca de la racionalidad de la fe. Pero ello implica, a su vez, tener que procurar un "horizonte sapiencial" que garantice la ansiada visión integral o unitaria del saber. En esta dirección precisa es donde hay que situar la solicitud que la teología viene realizando a la filosofía en general, y a la fenomenología en particular. La teología exige también de esta ciencia el esfuerzo por devolver la dilapidada confianza en la capacidad de la razón humana y en el servicio que ella presta en la búsqueda de la verdad y del fundamento último de lo real. Porque si por algo se ha de caracterizar la filosofía es, justamente, por la búsqueda de la verdad de las cosas (veritas rerum) ${ }^{39} \mathrm{o}$ del conocimiento absoluto como fundamento y sentido de la realidad en cuanto tal. Verdad ( $\alpha \lambda \dot{\eta} \theta \varepsilon 1 \alpha)$ sería el nombre auténtico para el filosofar ${ }^{40}$ y la filosofía de tipo sapiencial que aquí se propone es aquella que no rechaza albergar en su interior la perenne aspiración metafísica en aras de un fundamento último y absoluto. ${ }^{41}$ Dicho de otro modo, el tipo de filosofía que a día de hoy busca la teología deberá comenzar profesando una confianza inquebrantable en la capacidad de la razón para trascender los datos empíricos e intentar llegar, en su anhelo de la verdad, a algo absoluto, último y fundante.

\footnotetext{
${ }^{35}$ Una voz autorizada a este respecto es la del teólogo Joseph Ratzinger que antes de convertirse en el papa Benedicto XVI, insistía ya en decir que "la crisis de la teología postconciliar no es sino una consecuencia derivada directamente del abandono de los fundamentos filosóficos" (Benedicto XVI, 2007, p. 116).

${ }^{36}$ Véase (Ratzinger 1979 y 1982, pp. 121-128 y p. 380 respectivamente).

${ }^{37}$ Véase (Müller, 2010b).

${ }^{38}$ Hago mía la expresión utilizada por Benedicto XVI en su encuentro con los representantes de la ciencia en el Aula Magna de la Universidad de Regensburg el 12 de septiembre de 2006: (Benedicto XVI, 2006, pp. 730s.).

39 «Studium philosophiae non est ad hoc quod sciatur quod hominess sesserint, sed qualiter se habeat veritas rerum» (Tomás de Aquino, De caelo et mundo, I, 22, n. 8). Si la filosofía, como búsqueda de las causas profundas de todas las cosas, es el lugar más apto para la síntesis del sublime saber humano, la verdad representa sin lugar a dudas el fin de todo el universo: (Tomás de Aquino, Summa contra Gentiles, I, 4).
}

${ }^{40}$ Véase (Ortega y Gasset, 1965, pp. 388ss.).

${ }^{41}$ Véase (García Baró, 1993, p. 17). 
Pues bien, es en este horizonte sapiencial que acabamos de determinar donde la fenomenología estaría llamada a desempeñar su importantísima misión respecto a la teología, a saber, la de prestar una asistencia filosófica capaz de vertebrar sólidamente la búsqueda de lo verdadero, lo bueno y lo bello desde una dimensión sapiencial y metafísica. ${ }^{42}$ La fenomenología de Husserl representa, en este sentido, un enfoque más que pertinente a la hora de concretar cómo ha de entrar Dios en la filosofía. ${ }^{43}$ No se trata, simplemente, de repetir el trillado planteamiento de Heidegger sobre la constitución onto-teo-lógica de la metafísica occidental. Para el maestro, el verdadero problema filosófico consiste más bien en averiguar el modo en el que la ciencia primera se ve concernida por la teología. Su interés se dirige a la relación entre fenomenología y teología, pero en cuanto problema filosófico. Desde esta perspectiva, la fenomenología deberá conservar, por una parte, la plena autonomía de la labor filosófica, pero sin tener por ello que desechar, por otro lado, el contenido más propio de la teología. En un manuscrito del verano de 1934, con anotaciones valiosas para nuestro tema, el maestro afirma, en efecto, la necesidad de una filosofía autónoma "que necesariamente llega a una teleología y una teología filosóficas como camino aconfesional hacia Dios (als inkonfessioneller Weg zu Gott)" (Husserl, 2013, p. 259). En al apartado anterior, tuvimos ya la oportunidad de asomamos a este tipo de planteamientos teleologistas en relación con la teología formulados reiteradamente por el maestro. Lo que ahora me interesa, sin embargo, no es analizar este problema concreto sino el de la relación entre teología y fenomenología en cuanto problema filosófico intentando descubrir, sobre todo, la fecundidad y actualidad de dicha relación.

Es evidente que la fenomenología se presenta a los ojos de un Husserl maduro como la herramienta que permite hacer comprensible por qué la fe -en cuanto comunicada temporalmente a través de representaciones históricas y en el lenguaje concreto de un determinado tiempo- puede presentarse legítimamente con pretensión de verdad absoluta, a pesar, incluso, de la pluralidad de manifestaciones religiosas y de las no menos diversas interpretaciones filosóficas. La primera y más esencial aportación de la fenomenología a la teología sería, por tanto, la de fundamentar

\footnotetext{
${ }^{42}$ Véase (Juan Pablo II, 1993, p. 1169 y 1999, 6). Se trata de la clásica doctrina de la conversio tota de los trascendentales en cuanto coextensivos y atributos de un mismo Ens: Verum, bonum et pulchrum inter se convertuntur.

${ }^{43}$ Fue Heidegger quien puso en circulación el término onto-teo-logía de origen kantiano para caracterizar la forma metafísica de la filosofía occidental determinada por las cuestiones de Dios y del mundo. Esta filosofía, según el pensador de Todtnauberg, habría llegado a su consumación y también a su remate final con Nietzsche. Véase (Heidegger, 2006, pp. 31-68). Para Emmanuel Levinas, en cambio, el ultraje no se comente con el Ser ni es Dios quien ocupa su lugar, sino que la blasfemia consiste, al contrario, en la contaminación de Dios por el ser. Véase (Levinas, 1974 y 1993, p. 10 y pp. 137-260 respectivamente). En esta misma dirección, crítica con la idolatría conceptual que supone la noción hedeggeriana de ser, es en la que Jean Luc Marion ha formulado también su invitación a pensar a Dios sin el ser. Véase (Marion, 1991)
} 
racionalmente la irrenunciable aspiración a la verdad del discurso teológico. Esta tarea es ya, de por sí, suficientemente relevante para justificar la disposición favorable que el actual discurso teológico ha de tener respecto a la fenomenología. Husserl, sirviéndose del ejemplo de la filosofía y teología aristotélicas, llega a decir, incluso, que en el desarrollo in infinitum de ambas disciplinas podemos ver cómo la filosofía se proyecta cada vez de manera más concreta y la teología, a su vez, de manera más filosófica. Hasta el punto -llega a decir- de poder hablar de una esencial coincidencia entre ambas ${ }^{44}$. Pero esta convergencia-coincidencia de filosofía y teología no deja de ser, en realidad, sino la propuesta fundamental que hemos venido defendiendo desde el inicio mismo de este trabajo.

Cuando aquí proponemos la posibilidad de una teología de inspiración fenomenológica lo hacemos teniendo en cuenta las muchas aportaciones que la fenomenología puede prestar al pensamiento teológico. Y aunque mi objetivo se ciña exclusivamente a la fecundidad teológica de la fenomenología de Husserl sensu stricto, no es posible dejar de mencionar los numerosos y valiosísimos intentos realizados por muchos de sus discípulos que decidieron caminar en esta misma dirección. Es un hecho indudable, en efecto, que la fenomenología estuvo, in status nascendi, estrechamente vinculada a la teología. ${ }^{45}$ Ahí está, para corroborar nuestra afirmación, la obra, no suficientemente destacada ni valorada, de muchos de los discípulos de la primerísima hora que no dudaron en seguir al maestro y su enseñanza a la altura, al menos, de las Investigaciones Lógicas (Husserl, 1900, pp. 16-258 y 1901, pp. 6-529 y 537-775). Prácticamente todos los miembros del Círculo de Gotinga manifestaron un apego especial por los asuntos teológicos fundando la llamada "corriente realista" de la fenomenología, una tendencia que ha resultado ser especialmente fecunda para la filosofía de

44 "Im Unendlichen decken sich Philosophie (die immer konkreter werdende) und Theologie, die immer philosophischer werdende." (Husserl, 2013, p. 260).

${ }^{45}$ Merece la pena destacar a este respecto la obra de Jean Héring publicada en 1926 Fenomenología y filosofía religiosa (Héring, 1926). Lo que aquel joven profesor de la facultad protestante de teología en Estrasburgo -considerado por el mismo Husserl como "el más querido y fiel de [sus] antiguos alumnos"(Husserl, 1994, p. 253)- se propuso como objetivo para su tesis fue tratar de demostrar los grandes beneficios que la fenomenología podía suministrar a la filosofía religiosa. La pretensión que le animaba era, por una parte, rechazar la teología positivista de Karl Barth y esquivar, de otro lado, el irracional subjetivismo psicologista de Schleiermacher. Pero lo relevante para nosotros de esta obra, pionera en la difusión del movimiento fenomenológico en Francia, está en que ella proponía, desde su mismo umbral, la vinculación intrínsecamente respectiva entre Fenomenología y Teología. La convicción de Héring era que la epistemología husserliana ofrecía el fundamento necesario para que la teología moderna pudiera lograr unas de sus máximas aspiraciones, a saber, la de "aceptar de manera fiel la duda cartesiana como punto de partida y juez de su solidez” (Héring, 1926, pp. 130s.) y (Herrero-Hernández \& Hernández Marcelo, 2019, p. 111). 
la religión y la teología filosófica hasta el día de hoy. ${ }^{46} \mathrm{Y}$ otro tanto habría que decir de la recepción francesa de la fenomenología que, en sus diferentes fases y procesos, no ha cejado en su empeño de proseguir su propia andadura arropado siempre por el clima fenomenológico. ${ }^{47}$

Dicho esto, estamos en condiciones de decir ya, claramente, que la vinculación entre la fenomenología de Husserl y la teología, más allá de las vicisitudes históricas que haya atravesado dicho movimiento en sus diferentes fases y lugares, no se debió a ningún hecho casual ni obedeció tampoco a una afición particular del maestro por la temática teológica. Más aún, si la inspiración fenomenológica fue y sigue siendo especialmente fecunda para la teología lo es por mor de la propia fenomenología, sin que ésta tenga que forzar ninguno de sus presupuestos fundamentales para prestar tal servicio. La posibilidad para una fundamentación fenomenológica de la teología está ya dada, en efecto, en la consideración que esta ciencia de los fenómenos realiza sobre su mismo objeto. Porque si algo ha dicho la fenomenología es que su objeto, contra lo que podría esperarse a primera vista, no está formado por los fenómenos, sino por la fenomenalidad pura o la donación en cuanto tal. Y aunque su primer principio sea el de ira a las cosas mismas ( $Z u$ den Sachen selbst!), esto no significa sino atender aquello que nos da el modo de acceso inmediato a esas cosas, o sea, su aparecer. Como dice el mismo Husserl, la fenomenología se interesa sobre todo por "objetos en el cómo (Gegenstände im Wie)"48 de su aparecer. Y es precisamente ese centrar la mirada en el "cómo (Wie)" de la donación, el dato que resulta particularmente valioso para un pensar teológico que tiene a la analogía como la forma menos inadecuada para hablar del misterio. ${ }^{49}$

Creo haber justificado con lo dicho porqué la fenomenología puede convertirse en una excelente inspiración para la teología. Por un lado, aporta el horizonte sapiencial en el que la razón, sin claudicar de su capacidad y autonomía,

${ }^{46}$ Con Adolf Reinach a la cabeza, Edith Stein, Theodor Conrad, Hans Lipps, Alexandre Koyré, Jean Hering, Dietrich von Hildebrand, Hedwig Martius, Roman Ingarden, Moritz Geiger y el propio Max Scheler junto con los representantes más actuales: Josef Seifert, Fritz Wenisch, John Crosby, Miguel García Baró, Agustín Serrano de Haro, Pilar Fernández Beites o Rogelio Rovira.

${ }^{47}$ Figuras del pensamiento tan emblemáticos como la Paul Ricoeur, Merlau-Ponty, Emmanuel Levinas o Michel Henry. A ellos le siguieron la segunda generación de filósofos completamente consolidada, como J. Barash, R. Barbaras, R. Brague, J. Colette, J. L. Chrétien, J.-F. Courtine, F. Dastur, E. Escoubas, D. Frank, J.-Y. Lacoste, J.-L. Marion, M. Richir, P. Rodrigo. Y en su tercer momento a N. Depraz, E. Falque, J.-C. Gens, V. Houillon, J.-C. Lavigne, J. Poirée, C. Romano, F. Sebbah, Jocelyn Benoist, Jean Greisch. Para la contribución francesa al desarrollo de la fenomenología en la primera mitad del siglo XX véase (Ströker \& Janssen, 1989, pp. 243-350). Sobre la recepción francesa de la fenomenología véase (Waldenfels, 1998), (Escoubas \& Waldenfels, 2000), (Greisch, 2002b).

${ }^{48}$ (Husserl, 1966, Beilage VIII zu § 39, p. 117).

${ }^{49}$ Desde Tomás de Aquino o Francisco Suárez hasta E. Przywara, H. U. von Balthasar y el mismo Karl Rahner, son incontables los teólogos que han sostenido la necesidad de la analogia entis como presupuesto de la analogia fidei. Para la historia de la analogía véase (Przywara, 1996), (Sanabria, 1997, pp. 47-104) y (Ashworth, 2008). Véase también (Courtine, 2005), (Domínguez Prieto, 2009), (Long, 2011), (Seguró, 2015), (Floucat, 2016) y (Beuchot, 2017). 
ofrece la posibilidad de avanzar en la búsqueda de la verdad y del fundamento de lo real. La fenomenología, por otra parte, se presenta a sí misma como la ciencia de la fenomenalidad pura interesándose por el aparecer en cuanto aparecer, o sea, por la manera en que se da o se manifiesta la propia donación. Y es aquí, justamente, donde la teología encuentra un anclaje apropiado para elaborar su discurso, siempre analógico, sobre el misterio absoluto. Lo que finalmente habría que diseñar son las tareas concretas que la fenomenología tendría que realizar en su uso teológico. Nos limitaremos, no obstante, a enumerar únicamente las dos que consideramos más esenciales y urgentes: ${ }^{50}$

La primera de estas tareas consistiría en desarrollar una filosofía fenomenológica de la religión que ayudase a comprender de manera más adecuada la esencia de la vivencia religiosa a partir de su sentido interior, es decir, en cuanto vivencia intencional del sujeto. La reducción trascendental permitiría, en este sentido, fundamentar la constitución del sentido de los fenómenos religiosos. No se trata solo de describir tales fenómenos o vivencias, sino de profundizar en el lugar originario, sui generis, que ocupa la religión en el despliegue de la conciencia. Y por ello, necesariamente, tendrá que abordar también el problema de la relación con la idea de Dios. La filosofía fenomenológica de la religión deberá estar vinculada, expresa y resueltamente, con la metafísica.

La segunda tarea tendrá la difícil misión, por tanto, de elaborar una teología filosófica de inspiración fenomenológica. Deberá profundizar en el modo en el que Dios se da al ego trascendental. En principio, el marco para este quehacer seguiría siendo el que el maestro ha previsto a modo de regla, a saber: la necesidad de que toda trascendencia únicamente haya de constituirse en la vida de la conciencia sin que pueda, además, ser separada de ella. ${ }^{51}$ Es la dinámica de la "trascendencia en la inmanencia" la que impondría el tratamiento habitual de carácter éticoteleológico para resolver el problema de Dios al modo kantiano, es decir, como ideal trascendental, concepto límite del sentido del mundo. Y, sin embargo, el mismo

\footnotetext{
${ }^{50}$ Son numerosos los esfuerzos por concretar las aportaciones que la fenomenología puede hacer a la teología. A modo de ejemplo, véanse la síntesis de (Greisch, 2002a, pp. 17-70) y las contribuciones que se recogen en (Sönding \& Held, 2009). La teología católica, por su parte, ha realizado también algunos intentos valiosos de fundamentación metafísica para sí misma. Mencionamos especialmente la línea emprendida por el tomismo transcendental de Maréchal para refundar la metafísica y la filosofía de la religión. Como fruto más representativo de esta interpretación antropológico-trascendental de la filosofía escolástica, véase la obra temprana de Karl Rahner, Oyente de la Palabra (Rahner, 1997), que debe ser interpretada esencialmente desde el influjo ejercido por la tentativa de su maestro Erich Przywara por construir una Filosofía de la religión para la Teología católica a partir de su concepto central: la analogía entis. Véase (Przywara, 1962). Véase también (Ratzinger, 1985), (Pannemberg, 1996), (Müller, 2006, 2008 y 2010a y 2010b), (Dietzch \& Frigo, 2006), (Capelle-Dumont, 2011), (Falque, 2013) y (Dalferth, \& Hunziker, 2014).

${ }^{51}$ Véase (Husserl, 1991, p. 97): “(...) daß es das Bewußtseinsleben allein ist, in dem jedes Transzendente als von ihm Unabtrennbares sich konstituiert".
} 
Husserl ha contemplado la posibilidad de ir más allá de la prevista neutralización de la transcendencia de Dios. ${ }^{52}$ Son varios los pasajes de las obras mayores en los que Husserl, en efecto, parece haber abierto la puerta a la existencia de un ser "divino" o de una trascendencia que desborda la conciencia absoluta; de un Absoluto distinto del absoluto de la conciencia, es decir, de una trascendencia en un sentido diferente del todo a lo trascendente del mundo. ${ }^{53}$ Este tipo de afirmaciones serían, pues, las que habría que investigar más a fondo en una teología fenomenológica que tome en serio la tarea de pensar la cuestión de Dios como "el Logos absoluto, la verdad absoluta en el sentido pleno y total, como el unum verum bonum, hacia el que finalmente se dirigen, con enconado esfuerzo, todos y cada uno de los seres finitos en unidad". ${ }^{54}$

\section{Conclusiones}

Una comprensión de la fenomenología en cuanto fundamento filosófico para la teología como la que aquí he tratado de proponer implica admitir, de entrada, que entre razón y verdad existe una relación intrínsecamente respectiva. Significa reconocer, por una parte, que la razón -en cuanto estructura humana para aprehender la realidad desde su Logos interno- ha de esforzarse continuamente por alcanzar la verdad; y aceptar, por otro lado, que la verdad -interpretada como vivencia de la esencia real de las cosas, de aquello que le da el poder último de ser realidad, como dice Zubiri- ${ }^{55}$ que esa verdad, digo, no se descubre plenamente sino en la libre manifestación de Dios. Supone, en definitiva, asumir que para poder pensar $\mathrm{y}$ argumentar teológicamente hay que realizar previamente un discernimiento adecuado y riguroso de las posibilidades humanas de conocer la realidad. Pues bien, la fenomenología, tal como la hemos enunciado, representa uno de los caminos filosóficos más aptos para fundamentar este quehacer teológico más autoconsciente.

Como hemos tenido oportunidad de insistir, el principio básico en el que la fenomenología de Husserl ha intentado fundamentarse es, precisamente, el que afirma la validez universal de los juicios filosóficos. Esta validez universal no es sino la consecuencia, a su vez, de la confianza absoluta que el movimiento fenomenológico ha depositado, desde el inicio de su marcha, en la capacidad de la

\footnotetext{
${ }^{52}$ El título del parágrafo 58 del libro I de Ideas es significativo a este respecto, dice: "La trascendencia de Dios neutralizada (Die Traszendenz Gottes ausgeschaltet)" (Husserl, 1975, pp. 124s.)

${ }^{53}$ Véase (Husserl, 1975, p. 125). Se trata, sin duda, de una realidad absoluta y ésta "no es nada puesto ni afirmado a la buenaventura, sino un dato absolutamente indubitable -en un sentido adecuadamente ampliado del término darse (Unterschiede neuartiger Gegebenheitsmodi)" (Husserl, 1975, p. 185.

54 "Es ist der absolute Logos, die absoluteWahrheit im vollen und ganzen Sinn als das unum verum bonum, auf die alles endlich Seiende in der Einheit alles und jedes endlich Seiende umfangenden Strebens hin gerichtet ist" (Husserl, 2013, p. 250).
}

${ }^{55}$ Véase (Zubiri, 1999) y (Gracia, 2007). 
razón. La fenomenología, en efecto, consiste en la apuesta denodada por una razón universal que reclama, al mismo tiempo, un riguroso y esforzado ejercicio metódico hasta el punto de convertirse, finalmente, en el solar para el diálogo interdisciplinar con las demás ciencias. Desde esta perspectiva, una teología de inspiración fenomenológica podrá presentar, por su parte, su pretensión de verdad universal de una manera mucho más verosímil si cuenta con la asistencia básica de tal compañera filosófica. Porque la fenomenología, al introducir una fundamentación filosófica transcendental, otorga a la teología un recurso más que suficiente para desmontar todo cuestionamiento naturalista o materialista sobre la posibilidad misma de la idea de trascendencia. La fenomenología se anuncia, pues, como la aliada más acertada a la hora de reclamar el sentido y el significado de la trascendencia frente a cualquiera de los reduccionismos al uso, porque lo que ella dice, fundamentalmente, es que no hay hechos, sino fenómenos de diferentes tipos que se dan a la conciencia. Describirlos y encontrar su sentido normativo es, precisamente, la labor principal que ella pretende realizar.

\section{BIBLIOGRAFIAA}

Adorno, Th. W. (1996). Negative Dialektik. Jargon der Eigentlichkeit. In Tiedemann, Rolf (Hrsg.). Gesammelte Schriften. Band 6. Frankfurt am Main: Suhrkamp.

Ales Bello, Ángela. (1985). Husserl. Sul problema di Dio. Roma: Studium.

Ales Bello, Ángela. (1995). Teleo-logía y Teo-logía en Edmund Husserl. In Anuario Filosófico 28, pp. 11-18.

Ales Bello, Ángela. (2005). Edmund Husserl. Pensare Dio, credere in Dio. Padova: Messaggero.

Ales Bello, Ángela. (2009). The Divine in Husserl and Other Explorations. Dordrecht: Springer.

Alliez, Éric. (1995). De l’impossibilité de la phénoménologie. Sur la philosophie française contemporaine. Paris: Vrin.

Ashworth, E. Jennifer. (2008). Les théories de l'analogie du XIIe au XVIe siècle: Paris: J. Vrin. Benedicto XVI. (2006). Glaube, Vernunft und Universität. Erinnerungen und Reflexionen. In Acta Apostolicae Sedis, 98, pp.728-739.

Benedicto XVI. (2007). L'unità di missione e persona nella figura di Giovanni Paolo II. In Ratzinger, Joseph. Giovanni Paolo II. Il mio amato predecessore. Ciudad del Vaticano: Cinisello Basalmo. 
Benoist, Jocelyn. (1991). Husserl au-delà de l'onto-théologie?. In Les études philosophiques 4, pp. 433-458.

Benoist, Jocelyn. (1994). Vingt ans de phénoménologie française. In Mabin, Yves (dir.). (1996). Philosophie contemporaine en France (pp. 27-51) Paris: Ministère des Affaires Etrangères.

Beuchot, Mauricio. (2017). La analogía en la filosofía tomista reciente. México: Universidad Pontificia de México.

Cabria Ortega, José Luis. (2008). Dios, palabra, realidad. Filosofía y teología al encuentro. Santa Cruz de Tenerife: Idea.

Capelle-Dumont, Philippe. (2009a). Anthologie 1: Philosophie et théologie dans la période Antique. Alexandre, Jérôme (dir.). Paris: Cerf.

Capelle-Dumont, Philippe. (2009b). Anthologie 2: Philosophie et théologie au Moyen âge. Boulnois, Olivier (dir.). Paris: Cerf.

Capelle-Dumont, Philippe. (2010). Anthologie 3: Philosophie et théologie à l'époque moderne. Bardout, Jean-Christophe (dir.). Paris: Cerf.

Capelle-Dumont, Philippe. (2011). Anthologie 4: Philosophie et théologie à l'époque contemporaine. Greisch, Jean \& Hébert, Geneviève (dirs.). Paris: Cerf.

Caputo, John D. (2006). Philosophy and theology. Nashville: Abingdon.

Copertino, Giorgio Nicola. (2014). Interioritá e responsabilitá. Un itinerario a Dio tra Husserl, Stein e Levinas. Roma: Carmelitane.

Courtine, Jean-François. (2005). Inventio analogiae. Métaphysique et ontothéologie. Paris: Vrin.

Dalferth, Ingolf U. \& Hunziker, Andreas (Hrsgs.). (2014). Gott denken-ohne Metaphysik? Zu einer aktuellen Kontroverse in Theologie und Philosophie. Tübingen: Mohr Siebeck.

Delgado, Mariano \& Vergauwen, Guido (Hrsg.). (2003). Glaube und Vernunft - Theologie und Philosophie. Aspekte ihrer Wechselwirkung in Geschichte und Gegenwart. Fribourg: Academic Press.

Dietzsch, Steffen \& Frigo, Gian Franco (Hrsgs.). (2006), Vernunft und Glaube. Ein philosophischer Dialog der Moderne mit dem Christentum. Père Xavier Tilliette SJ zum 85. Geburtstag. Berlin: Akademie Verlag.

Domínguez Prieto, Pablo. (2009). La analogía teológica. Su posibilidad metalógica y sus consecuencias físicas, metafísicas y antropológicas. Madrid: Publicaciones Universidad San Dámaso.

Dufrenne, Mikel. (1973). Pour une philosophie non théologique. In Id. Le Poétique. Paris: PUF, pp. 7-57.

Escoubas, Elian \& Waldenfels, Bernhard (Eds./Hrsg.) Phénoménologie française et phénoménologie allemande. Paris: L'Harmattan.

Falque, Emmanuel. (2013). Passer le Rubicon. Philosophie et théologie: essai sur les frontières. Bruxelles: Lessius. 
Fink, Eugen. (1968) Los conceptos operatorios en la fenomenología de Husserl. In Husserl. Troisième Colloque de Royaumont (1959). Paris: Minuit. Traducción de Podetti, A. Husserl. Tercer Coloquio Filosófico de Royaumont (pp. 192-205). Buenos Aires: Paidós.

Flores D’Arcais, Paolo. (2008). Once tesis contra Habermas. In Claves de razón práctica. 179 , pp. 56-60.

Flores d'Arcais, Paolo. (2014). Por una democracia sin Dios. Madrid: Trotta.

Floucat, Yves. (2016). Pour une métaphysique de l'être en son analogie. De Heidegger à Thomas d'Aquin. Paris: Lethielleux.

García Baró, Miguel. (1993). Ensayos sobre lo Absoluto. Madrid: Caparrós.

González de Cardedal, Olegario. (2003). Filosofía y teología. Revista de Occidente, 258, pp. 5-31.

González de Cardedal, Olegario. (2008). El quehacer de la teología. Génesis, estructura, misión. Salamanca: Sígueme.

Gracia, Diego. (2007). Voluntad de verdad. Para leer a Zubiri. Madid: Triacastela.

Greisch, Jean. (2002a). Le buisson ardent et les lumières de la raison. L'invention de la philosophie de la religión. Band II: Les aproches phénomenologiques et analytiques Paris: Cerf.

Greisch, Jean. (2002b). Les yeux de Husserl en France. Les tentatives de refondation de la phénoménologie dans la deuxième moitié du xxe siècle. In Dupont, Pascal \& Cournarie, Laurent (coord.) Phénoménologie: un siècle de philosophie (pp. 45-74). Paris: Ellipses.

Habermas, Jürgen. (2005). Vorpolitische Grundlagen des demokratischen Rechtsstaates? In Florian Schuller (Ed.), Dialektik der Säkularisierung: Über Vernunft und Religion. Freiburg; Basel; Wien: Herder.

Hailer, Martin. (2006). Glauben und Wissen. Arbeitsbuch Theologie und Philosophie. Göttingen: Vandenhoeck \& Ruprecht.

Harr, Michel. (1999). La philosophie française entre phénoménologie et métaphysyque Paris: Presses Universitaires de France.

Hart, James G. (1990). I, we and God: ingredients of Husserl's theory od community. In Samuel Ijsseling (Ed.), Husserl-Ausgabe und Husserl-Forschung (pp. 125-149). Dordrecht: Kluwer Academic.

Hegel, W. (1983). Vorlesungen über die Philosophie der Religion. B. 1, Einleitung. Der Begriff der Religion, edición de Walter Jaeschke. Hamburg: Mainer.

Hegel, W. (1986). Phänomenologie des Geistes. In Moldenhauer, E. \& Michel, H. M. (Hrsgs.). Hegel. Werke in zwanzig Bände. Band 3. Frankfurt: Surhkamp.

Hegel, W. (1989). Lecciones sobre la filosofía de la religión. Vol I: El concepto de religión. Traducción de Ricardo Ferrara. Madrid: Alianza Editorial.

Hegel, W. (2009). Fenomenología del espíritu. Edición y traducción de Manuel Jiménez Redondo. Valencia: Pretextos. 
Heidegger, Martin. (2006). Die onto-theo-logische Verfassung der Metaphysik. In Heidegger Gesamtausgabe. Band 11. Identität und Differenz. (1957, 2006) pp. 31-68.

Held, Klaus. (2010). Gott im Edmund Husserls Phänomenologie. In Ierna, C. \& al. (eds.). Philosophy, Phenomenology, Sciences. Essays in Commemoration of Edmund Husserl. (pp. 723-738). Dordrecht: Springer.

Héring, Jean. (1926). Phénoménologie et philosophie religieuse. Étude sur la théorie de la connaissance religieuse. Paris: Alcan.

Herrero-Hernández, Francisco-Javier (2016a). La crítica de Edmund Husserl a la idea de Dios y el giro teológico de la fenomenología. In Murillo Murillo, Ildefonso (Ed.). Pensar y conocer a Dios en el siglo XXI (pp. 233-243). Madrid: Diálogo Filosófico.

Herrero-Hernández, Francisco-Javier(2016b). Transcendencia y Postsecularidad. La cuestión de Dios en la cultura contemporánea. In Aporía. Revista Internacional de Investigaciones Filosóficas, 2016, volumen 12, pp. 4-22.

Herrero-Hernández, Francisco Javier \& Hernández-Marcelo, Jimmy. (2019). Jean. Héring. Fenomenología y Filosofía religiosa. Estudio sobre la teoría de la Conciencia religiosa. Traducción, introducción y notas. Postfacio de Sylvain Camilleri. Madrid: Universidad San Dámaso, 2019.

Holscher, Uvo. (1968). Anfängliches Fragen. Studien zur frühen griechischen Philosophie. Göttingen: Vandenhoeck \& Ruprecht.

Housset, Emmanuel. (2010). Husserl et l'idée de Dieu. Paris: Cerf.

Husserl, Edmund. (1900). Logische Untersuchungen. Erster Theil: Prolegomena zur reinen Logik. Text der 1. Und der 2. Auflage. In Holenstein, Elmar (Hrsg.). (1975). Husserliana. Edmund Husserl. Gesammelte Werke. Band XVIII. (pp. 16-258). Den Haag: Martinus Nijhoff.

Husserl, Edmund. (1901). Logische Untersuchungen. Erster Teil: Untersuchungen zur Phänomenologie und Theorie der Erkenntnis. Text der 1. Und der 2. Auflage ergänzt duch Annotationen ud Beiblätter aus dem Handexemplar. In Panzer, Ursula (Hrsg.). (1984). Husserliana. Edmund Husserl. Gesammelte Werke. Band XIX/1. (pp. 6-529). Den Haag: Martinus Nijhoff.

Husserl, Edmund. (1901). Logische Untersuchungen. Zweiter Teil: Elemente einer phänomenologischen Aufklärung der Erkenntnis. Text der 1. Und der 2. Auflage ergänzt duch Annotationen ud Beiblätter aus dem Handexemplar. In Panzer, Ursula (Hrsg.). (1984). Husserliana. Edmund Husserl. Gesammelte Werke. Band XIX/2. (pp. 533-775). Den Haag: Martinus Nijhoff.

Husserl, Edmund. (1954). Die Krisis der europäischen Wissenschaften und die tranzendentale Phänomenologie. Eine Einleitung in die phänomenologische Philosophie. In Biemel, W. von (Hrsg.). Husserliana. Edmund Husserl. Gesammelte Werke. Band VI. Den Haag: Martinus Nijhoff.

Husserl, Edmund. (1966). Zur Phänomenologie des inneren Zeitbewusstseins (1893-1917). In Boehm, Rudolf. (Hrsg.). Husserliana. Edmund Husserl. Gesammelte Werke. Band X. Den Haag: Martinus Nijhoff. 
Husserl, Edmund. (1969). Zur Phänomenologie des inneren Zeitbewusstseins (1893-1917). In Boehm, Rudolf. (Hrsg.). Husserliana. Edmund Husserl. Gesammelte Werke. Band X. Den Haag: Martinus Nijhoff.

Husserl, Edmund. (1974). Formale und transzendentale Logik. Versuch einer Kritik der logischen Vernunft. In von Janssen, Paul (Hrsg.). Husserliana. Edmund Husserl. Gesammelte Werke. Band XVII. Den Haag: Martinus Nijhoff.

Husserl, Edmund. (1975). Ideen zu einer reinen Phänomenologie und phänomenologischen Philosophie. Erstes Buch: Allgemeine Einführung in die reine Phänomenologie. In Schuhmann, Karl (Hrsg..). Husserliana: Edmund Husserl Gesammelte Werke Band III/1. Den Haag: Martinus Nijhoff.

Husserl, Edmund. (1976). Die Krisis der europciischen Wissenschaften und die transzendentale Phönomenologie. Eine Einleitung in die phenomenologishce Philosophie. In Biemel, Walter (Hrsg.). Husserliana. Edmund Husserl. Gesammelte Werke. Band VI. Den Haag: Martinus Nijhoff.

Husserl, Edmund. (1987). Aufsatze und Vorträge (1911-1921). Mit erganzenden Texten. In Nenon, Thomas \& Sepp, Hans Rainer (Hrsgs.). Husserliana. Edmund Husserl. Gesammelte Werke Edmund Husserl. Band XXV. Den Haag: Martinus Nijhoff.

Husserl, Edmund. (1989). Aufsätze und Vorträge (1922-1937). In Nenon, Thomas \& Sepp, Hans Rainer (Hrsgs.). Husserliana. Edmund Husserl. Gesammelte Werke. Band XXVII. Dordrecht - Boston - London: Kluwer Academic Publischers.

Husserl, Edmund. (1991). Cartesianische Meditationen und pariser Vortrage. In Strasser, S. (Hrsg.). Husserliana. Edmund Husserl. Gesammelte Werke. I. Dordrecht - Boston - London: Kluwer Academic Publischers.

Husserl, Edmund. (1994). Die Göttinger Schule. In Ijsseling, Samuel. (Hrsg.). Edmund Husserl. Husserliana. Dokumente. Band III. Briefwechsel. Teil 3. Dordrecht - Boston London: Springe.

Husserl, Edmund. (2013). Grenzprobleme der Phänomenologie. Analysen des Unbewusstseins und der Instinkterenzprobleme. Metaphysik. Späte Ethik. Texte aus dem Nachlass (1908-1937). In Sowa Rochus \& Vongehr, Thomas (Hrsgs.). Husserliana. Edmund Husserl. Gesammelte Werke. Band XLII. Dordrecht - Heidelberg - New York - London: Springer.

Iribarne, Julia Valentina. (1994): Dios en la fenomenología de Husserl. In Areté, Vol. VI, no 2, pp. 239-252.

Jaeger, Werner. (1953). Die Theologie der frühen friechischen Denker. Stuttgart: 1953. traducida al español por Gaos, José. (1952). La teología de los primeros filósofos griegos. México.

Jaeger, Werner. (1965). Cristianismo primitivo y paideia griega. México: Fondo de Cultura Económico.

Janicaud, Dominique. (1990). Le tournant théologique de la phénoménologie française. Paris: L'éclat.

Janicaud, Dominique. (1998). La phénomenologie éclatée. Paris: L’éclat. 
Janicaud, Dominique. (2000). Phénoménologie ou métaphysique? In Escoubas, Elian \& Waldenfels, Bernhard (Hrsgs.). Phénoménologie française et phénoménologie allemande (pp. 175-213). Paris: L’Harmattan.

Juan Pablo II, San. (1993). Veritatis splendor. Carta Encíclica sobre algunas cuestiones fundamentales de la enseñanza moral de la Iglesia (6 de agosto de 1993). In Acta Apostolicae Sedis, 85 .

Juan Pablo II, San. (1999). Fides et ratio. Carta Encíclica sobre las relaciones entre fe y razón (14 de septiembre de 1998). In Acta Apostolicae Sedis, 91.

Kant, Inmanuel. (1800). Logik. Ein Handbuch zu Vorlesungen. Herausgegeben von Gottlob Benjamin Jäsche. Königsberg, Friedrich Nicolovius, AA, IX.

Kant, Inmanuel. (1969). Die Religion innerhalb der Grenzen der bloßen Vernunft en Wilhelm Weischedel (Hrsg.), Immanuel Kants Werke, Band IV. Trad. de F. Martínez Marzoa (1986). La religión dentro de los límites de la mera razón. Madrid: Alianza.

Kant, Inmanuel. (1978). Der Streit der Fakultäten. In W. Weischedel (Hrsg.). Werkausgabe. Bd. XI. Frankfurt am Main; traducción de Rodríguez Aramayo, Roberto y estudio preliminar de Gómez Caffarena, José. (1999). La contienda entre las facultades de filosofía y teología. Madrid: Trotta.

Lacoste, J. Y. (1998). Philosophie-Théologie. In Id. (dir.), Dictionnaire critique de Théologie. Paris: PUF.

Laycock, Steven William \& Hart, James G. (Eds.). (1986). Essays in phenomenological theology. Albany: State University of New York Press.

Levinas, Emmanuel. (1961). Totalité et Infini. Essai sur l'extériorité. Den Haag: Martinus Nijhoff.

Levinas, Emmanuel. (1974). Autrement qu'être ou au-delà de l'essence. Den Haag: Martinus Nijhoff.

Levinas, E. (1988). La Blible et les Grecs. In id., À l'heure des nations. Paris: Les Éditions de Minuit.

Levinas, Emmanuel. (1993). Dieu, la mort et le temps. Paris: Grasset.

Levinas, Emmanuel. (1995). Altérité et transcendance. Paris: Fata Morgana.

Lo, Lee-Chun (2008). Die Gottesauffassung in Husserls Phänomenologie. Frankfurt am Main - New York: P. Lang cop.

Long, Steven A. (2011). Analogia entis. On the analogy of being, metaphysics, and the act of faith. Indiana: University of Notre Dame Press.

Marion, Jean-Luc. (1991). Dieu sans l'être. Paris: PUF.

Marion, Jean-Luc. (1992). De l'histoire de l'être' à la donation du posible. In Le Débat 72, pp. 167-176.

Müller, Klaus (2006). Glauben, Fragen, Denken. Band 1: Basisthemen in der Begegnung von Philosophie und Theologie. Münster: Aschendorff. 
Müller, Klaus (2008). Glauben, Fragen, Denken. Band 2 Weisen der Weltbeqiehung. Münster: Aschendorff.

Müller, Klaus (2010a). Glauben, Fragen, Denken. Band 3: Selbstbęiehung und Gottesfrage. Münster: Aschendorff.

Müller, Klaus (2010b). Per una teologia autocosciente. Lezioni su religion e modernitá. Lugano: Eupress FTL.

Ortega y Gasset, José. (1965). Origen y epilogo de la Filosofía. In Obras Completas, IX, Madrid: Revista de Occidente.

Pannenberg, W. (1996). Theologie und Philosophie. Ihr Verhältnis im Lichte ihrer gemeinsamen Geschichte. Göttingen: Vandenhoeck \& Ruprecht. Traducción de Fernández de Mururi Duque, Rafael. (2001). Una historia de la filosofía desde la idea de Dios. Salamanca: Sígueme. 2001.

Pannenberg, W. (1988). Das Ende der Mataphysik und der Gottesgedanke. In id. Metaphysik und Gottesgedanke. Göttingen: Vandenhoeck \& Ruprecht. Traducido por Abella, Manuel. (1999). Metafísica e idea de Dios. Madrid: Caparrós.

Przywara, Erich. (1962). Religionsphilosophie katholischer Theologie. In Schriften. Band II: Religionsphilosophische Schriften (pp. 376-512). Einsiedeln: Johannes-Verlag.

Przywara, Erich. (1996). Analogia entis. Metaphysik: Ur-Struktur und All-Rhythmus. In Schriften. Band III. Einsiedeln: Johannes Verlag.

Rahner, Karl. (1976). Grundkurs des Glaubens. Einfuhrung in den Begriff des Christentums. Freiburg/Basel/Wien: Herder.

Rahner, Karl. (1997). Hörer des Wortes. Zur Grundlegung einer Religionsphilosophie. In Karl-Rahner. Sämtliche Werke. Band 4. Freiburg im Breisgau: Herder.

Ratzinger, Joseph. (1979). Was ist Theologie? Rede zum 75. Geburtstag von Hermann Kardinal Volk. In Internationale katholische Zeitschrift Communio 8, pp. 121-128.

Ratzinger, Joseph. (1985). Teoría de los Principios teológicos. Materiales para una teología fundamental. Barcelona: Herder.

Rohls, Jan. (2002). Philosophie und Theologie in Geschichte und Gegenwart. Tübingen: Mohr Siebeck.

Sanabria, José R. (1997). Analogía y hermenéutica. In Mardones, José M. \& Sanabria, José R. (comps.). ¿Tiene la analogía alguna función en el pensar filosófico? (pp. 47-104). México: Universidad Iberoamericana.

Scheler, Max. (2007). Vom Ewigen im Menschen, GW 5 Studienausgabe. Bonn: Bouvier. Seguró, Miquel. (2015). Sendas de finitud. Analogía y diferencia. Barcelona: Herder.

Sönding, Thomas \& Held, Klaus. (2009). Phänomenologie und Theologie. Freiburg im Breisgau: Herder.

Strasser, S. Das Gottesproblem in der Spätphilosophie Husserls. In Philosophisches Jahrbuch, 67, pp.130-142. 
Ströker, Elisabeth \& Janssen, Paul. (1989). Phänomenologische Philosophie. Freiburg: Karl Albert.

Torres Queiruga, Andrés (2000). El problema de Dios en la modernidad. Pamplona: Estella.

Van Breda, Herman Leo. (1949). Husserl et le problème de Dieu. In Evert Willem Beth (Ed.), Proceedings of the tenth international congress of philosophy. Amsterdam: North Holland Publishing Corporation.

Waldenfels, Bernhard. (1998). Phänomenologie in Frankreich. Frankfurt am Main: Suhrkamp.

Walton, Roberto J. (2012). Husserl on Teleology and Theology. In Revista Estudios de Filosofía 45, pp. 81-103.

Weischedel, Wilhelm. (1998). Der Gott der philosophen. Grundlegung einer Philosophischen Theologie im Zeitalter des Nihilismus. Darmstadt: Primus.

Zubiri Apalategui, Xavier. (1999). El hombre y la verdad. Madrid: Alianza EditotorialFundación Xavier Zubiri. 